Medicine Updates

Faculty of medicine

April 2020,volume 1, issue 1 https://muj.journals.ekb.eg

dean@med.psu.edu.eg

vice_dean_postgraduate@med.psu.edu.eg

DOI:10.21608/muj.2020.25106.1007

ISSN : 2682-2741

Submitted: $3 / 3 / 2020$

Accepted : 4/3/2020

Pages:9-28

\title{
The Possible Antidiabetic Effects of Ranolazine Versus Gliclazide In HFD/STZ-Induced Type 2 Diabetes In Male Albino Rats
}

\author{
Shereen S. Elkholy (1), Mona K. Tawfik (2), Amira S. Mohammed (3)
}

Department of Pharmacology, Faculty of Medicine, Portsaid University, Portsaid (1), Department of Pharmacology, Faculty of Medicine, Suez Canal University, Ismailia, (2),(3), Egypt

\section{Abstract}

Background: Type 2 diabetes is a major illness that affects millions of people. The key causes of type 2 diabetes are insulin resistance and decreased insulin secretion

Objectives: To evaluate the possible effects of ranolazine versus gliclazide on blood glucose levels, HbA1c, nitric oxide and oxidative stress markers in HFD/STZ-induced type 2 diabetes in male albino rats and their effect on the histopathological picture of the pancreas and on apoptosis .

Materials and Methods: Thirty-two male albino rats were divided into four groups. The normal control group which received saline $(1 \mathrm{mg} / \mathrm{kg} /$ day) for 5 weeks. The diabetic control group that received saline $(1 \mathrm{mg} / \mathrm{kg} /$ day $)$ for 5 weeks. The gliclazide-treated group that received gliclazide $(10 \mathrm{mg} / \mathrm{kg})$ twice daily for 5 weeks and the ranolazine-treated group that received ranolazine (20 $\mathrm{mg} / \mathrm{kg}$ ) twice daily for 5 weeks. Body weight and blood glucose levels were measured weekly for the 5 weeks, then blood samples were obtained for various biochemical analysis: lipid profile, HbA1c, AGEs and NO. Then rats were sacrificed and the pancreatic tissues were obtained for oxidative stress markers 
estimation, for histopathological examination using haematoxylin and eosin stain and for estimation of caspase-3 marker.

Results: Ranolazine improved diabetes by reducing fasting blood glucose level, $\mathrm{HbA1c}$, NO and AGEs. Moreover, it improved the oxidative stress markers, the histopathological picture of the pancreas and decreased the apoptosis.

Conclusion: Ranolazine has the potential to become a novel agent for treating type 2 diabetes patients .

Keywords: Diabetes mellitus, STZ, Oxidative stress markers, Caspase-3, HbA1c.

\section{$\underline{\underline{\text { Introduction }}}$}

Type 2 diabetes is a major illness that affects millions of people. The chief causes of type 2 diabetes are insulin resistance and decreased insulin secretion[1]. About 2,623,000 people in Egypt are affected, with the expectation of 6,726,000 people in 2030 [2]. Moreover, Type 2 diabetes is considered a risk factor for cardiovascular events and is a substantial predictor of cardiovascular morbidity and mortality [3].

Ranolazine is a novel drug for angina that decreases frequency of angina attacks and recovers exercise tolerance in the affected patients [4]. Ranolazine is a selective inhibitor of cardiac late sodium channels that result in decreasing the intracellular $\mathrm{Na}^{+}$and $\mathrm{Ca}^{+2}$, leading to its anti-anginal properties in myocardial ischemia. [5]. Ranolazine also has been revealed to lower $\mathrm{HbA}_{1 \mathrm{c}}$ in cardiac patients with comorbid diabetes [6]. In the Combination Assessment of Ranolazine In Stable Angina trial, ranolazine significantly lowered $\mathrm{HbA1c}$ levels by $0.7 \pm 0.18 \%$ when it is given for 12 weeks irrespective of concomitant oral antidiabetic therapy. Furthermore, long-term ranolazine treatment shows preservation of $\beta$-cell and improvement of insulin secretion [7]. 
Gliclazide is a standard drug that used frequently for type 2 diabetes mellitus (DM) treatment. The drug stimulates the pancreatic $\beta$-cells to release insulin resulting in reduction of blood glucose level. Moreover, gliclazide has antioxidant and extra - pancreatic properties [8].

Linking the fact that calcium channel antagonists and $\beta$-receptor blockers produce hyperglycmia and that ranolazine behaves as an add on treatment for beta-antagonists and calcium channel antagonists in anginal patients with the fact that ranolazine has been revealed to lower $\mathrm{HbA}_{1 c}$ in patients with angina, ranolazine was logical applicant for study. Therefore, the current study was conducted to observe the possible antidiabetic effect of ranolazine and its mechanisms in HFD/STZ- induced type 2 diabetes in rats.

\section{Materials and Methods}

Experimental animals: Thirty-two adult male albino rats, weighing 180-200 g. They were accommodated in polyethylene cages with free access to to standard animal diet or HFD and tap water ad libitum. The rats were kept under standard conditions of normal light-dark cycle and temperature adjusted between $25-30^{\circ} \mathrm{C}$.

Induction of type-2 diabetes: Rats were fed with a high fat diet (HFD) for four weeks. Followed by receiving a single injection of STZ (30 mg/kg, i.p.) [9]. Three days after STZ injection, rats with fasting blood glucose levels $>200 \mathrm{mg} / \mathrm{dl}$ were designated as diabetic [10]. Then, rats divided into four groups of eight animals each. The normal control group that received saline $(1 \mathrm{mg} / \mathrm{kg} /$ day) for 5 weeks. The diabetic control group that received saline $(1 \mathrm{mg} / \mathrm{kg} / \mathrm{day})$ for 5 weeks. The gliclazide-treated group that received oral gliclazide $(10 \mathrm{mg} / \mathrm{kg}$ ) twice daily for 5 weeks [11]. The ranolazine-treated group that received oral ranolazine $(20 \mathrm{mg} / \mathrm{kg})$ twice daily for 5 weeks [12]. 
Body weights and blood glucose levels were measured weekly for the 5 weeks. Insulin resistance was assessed using the homeostasis model assessment for insulin resistance (HOMA-IR) index. At the end of 5 weeks treatment, blood samples were obtained for various biochemical analysis: HbA1c, Lipid profile (Total cholesterol, HDL, TG, LDL and VLDL), advanced glycated endproducts (AGEs) and nitric oxide (NO). Then rats were sacrificed and The pancreatic tissues were gained for oxidative stress markers (MDA, SOD, GSH, CAT) estimation, for histopathological examination using hematoxylin and eosin (H and $\mathrm{E}$ ) stain and for caspase-3 marker assessment by immunohistochemical staining. Assessment of caspase 3 staining using semiquantitative method: The H score. An H-score was attained between 0 and 300 where 300 was equivalent to $100 \%$ of islets cells of pancreas stained strongly $(3+)$ [13].

Statistical analysis: All the grouped data were statistically estimated using statistical package for social sciences (SPSS) program (windows version number 10) and were expressed as mean \pm SEM. The gained data were analyzed by one-way ANOVA followed by Banferroni's multiple comparisons test. Data with a $\mathrm{P}$ value $<0.05$ were considered statistically significant.

\section{$\underline{\underline{\text { Results }}}$}

Fig (1) Revealed that HFD/STZ-challenged rats showed significant surge in FBS levels in comparison to the normal control group $(\mathrm{p}<0.05)$ beginning from the start of the study and continued till the end of experiment. Treatment with either gliclizide or ranolazine significantly $(\mathrm{p}<0.05)$ lowered FBS levels as compared to diabetic control group; with significant $(\mathrm{p}<0.05)$ differences between the two treated groups.

Fig (2) and Table (1) Revealed that HFD/STZ-challenged rats showed significant reduction in BW in comparison with normal control group $(\mathrm{p}<0.05)$ starting from the fourth week. Treatment with gliclizide significantly $(\mathrm{p}<0.05)$ elevate BW as compared to diabetic control group starting from the third week. 
Treatment with ranolazine significantly $(\mathrm{p}<0.05)$ rise $\mathrm{BW}$ as compared to diabetic control group starting from the second week; with significant $(\mathrm{p}<0.05)$ differences between the two treated groups.

Table (2) Revealed that HFD/STZ-challenged rats showed a significant augmentation in cholesterol, LDL, TG, and VLDL levels accompanying by significant reduction in HDL levels compared to normal-control group $(\mathrm{p}<0.05)$. Treatment with either gliclizide or ranolazine significantly mitigated these parameters as compared to diabetic-control group $(\mathrm{p}<0.05)$ with significant $(\mathrm{p}<0.05)$ differences between the two treated groups in LDL only.

Fig (3) Showed that HFD/STZ-challenged rats was associated with significant $(\mathrm{p}<0.05)$ surge in $\mathrm{HbA}_{1 \mathrm{c}}$, HOMA, AGEs, and $\mathrm{NO}$ levels in comparison with the normal control group. Treatment with either gliclizide or ranolazine significantly $(\mathrm{p}<0.05)$ ameliorated these augmented levels compared to diabetic control group with insignificant $(p>0.05)$ differences between the two treated groups except on HOMA and NO level where treatment with ranolazine significantly $(\mathrm{p}<0.05)$ decreased HOMA and NO level in comparison with gliclizide treated group.

Fig (4) Showed that HFD/STZ-challenged rats increased oxidative stress in the form of significant $(\mathrm{p}<0.05)$ augmentation of MDA level and significant $(\mathrm{p}<0.05)$ decrease in GSH, SOD and CAT levels compared to normal control group. These lethal effects were significantly $(\mathrm{p}<0.05)$ improved with either gliclizide or ranolazine as compared to diabetic control group with insignificant $(p>0.05)$ differences between the two treated groups on MDA level. Clearly, treatment with ranolazine revealed significant difference $(\mathrm{p}<0.05)$ in GSH, SOD and CAT levels compared to gliclizide treated group.

Fig (5A-D) Examination of $\mathrm{H}$ and $\mathrm{E}$ stained islets of pancreas exhibited that: In normal-control group, there was preserved rounded contour of islets (C), the cells have eosinophilic cytoplasm, some are arranged in acinar pattern (Fig-5A). On the other hand, pancreatic islets cells from diabetic-control group 
showed lost rounded contour of islets with islets shrinkage due to reduction in the number of cells within each islet. The cells have eosinophilic cytoplasm, rounded to angulated nuclei and lost acinar pattern with shrunken cells size. Most of cells showed vacuolar (hydropic) degeneration (V), with scattered deeply stained eosinophilic bodies (apoptotic bodies) (A) (Fig-5B). Treatment with gliclizide showed focally restored contour of some islets, others still have irregular contours and less cellular due to an increase in the number of cells within each islet. The cells have eosinophilic cytoplasm, rounded to angulated nuclei, still lost acinar pattern and residual vacuolar (hydropic) degeneration, with apoptotic bodies (Fig-5C). Treatment with ranolazine showed restored rounded contour of islets; having cellular islets due to an increase in the number of cells within each islet. The cells have eosinophilic cytoplasm, rounded regular nuclei, restored acinar pattern, no vacuolar (hydropic) degeneration, with very few apoptotic bodies (Fig-5D).

Fig 6(I,II) Revealed that in normal-control group, pancreatic islets cells showed no staining, or very weak staining (Fig 6-IA). On the other hand, pancreatic islets cells from diabetic-control group showed moderate to marked staining (Fig 6-IB) with significant $(\mathrm{p}<0.05)$ augmentation of caspase-3 level in comparison with the normal control group (Fig 6-II). Treatment with either gliclizide or ranolazine showed decreased staining(Fig 6-IC,ID) with significant $(\mathrm{p}<0.05)$ decrease in caspase-3 level in comparison with diabetic control group (Fig 6-II). Treatment with ranolazine significantly $(\mathrm{p}<0.05)$ reduced caspase-3 level compared to gliclizide treated group(Fig 6-II).

Figure 1: Effect of Gliclizide and Ranolazine on FBG (mg/dl) levels in HFD and low dose STZ-induced diabetic rats: 


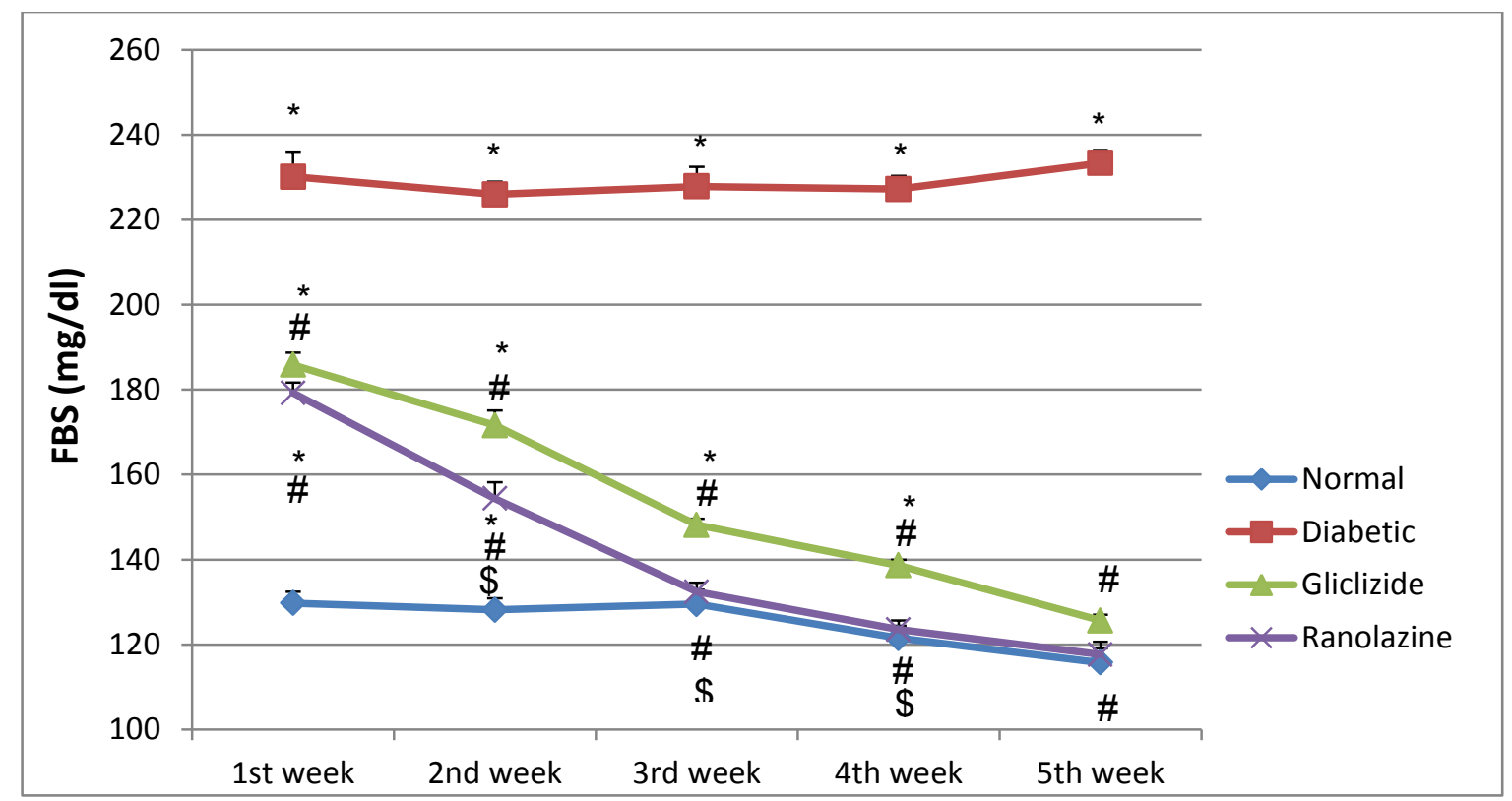

*Statistically significant difference versus normal control group (P-value $<0.05)$ \# Statistically significant difference versus diabetic control group (P-value $<0.05)$ $\$$ Statistically significant difference versus gliclazide-treated group $(P$-value $<0.05)$

Figure 2: Effect of Gliclizide and Ranolazine on body weight (BW) (grams) in HFD and low dose STZ-induced diabetic rats:

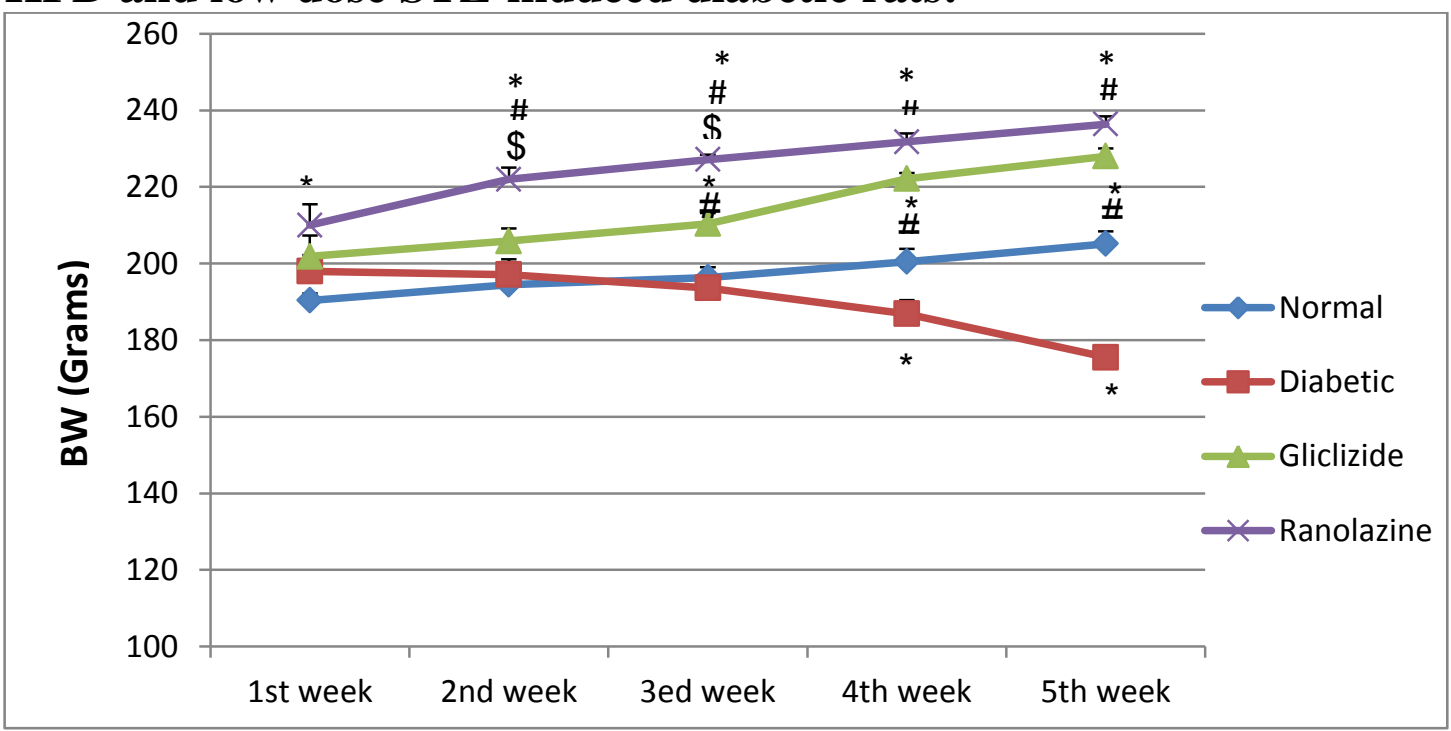

*Statistically significant difference versus normal control group $(P$-value $<0.05)$ \# Statistically significant difference versus diabetic control group $(P$-value $<0.05)$ $\$$ Statistically significant difference versus gliclazide-treated group $(P$-value $<0.05)$ 
Table 1: Body weight of all experimental groups at the end of the study:

\begin{tabular}{|l|c|}
\hline & $\begin{array}{c}\text { Body weight }(\mathrm{g}) \\
\text { (Mean } \pm \text { SEM) }\end{array}$ \\
\hline Normal control & $205 \pm 3.3$ \\
\hline Diabetic control & $176 \pm 2^{*}$ \\
\hline Gliclizide & $228 \pm 2.1^{* \#}$ \\
\hline Ranolazine & $236 \pm 2.1^{* \#}$ \\
\hline
\end{tabular}

*Statistically significant difference versus normal control group $(P$-value $<0.05)$

\# Statistically significant difference versus diabetic control group $(P$-value $<0.05)$

$\$$ Statistically significant difference versus gliclazide-treated group $(P$-value $<0.05)$

Table 2: Comparison of lipid profile (cholesterol (mg/dl), TG (mg/dl), HDL (mg/dl), LDL (mg/dl) and VLDL(mg/dl)) among the experimental groups.

\begin{tabular}{|c|c|c|c|c|c|}
\hline & $\begin{array}{c}\text { Cholesterol } \\
(\mathrm{mg} / \mathrm{dl})\end{array}$ & $\begin{array}{c}\text { TG } \\
(\mathrm{mg} / \mathrm{dl})\end{array}$ & $\begin{array}{c}\text { HDL } \\
(\mathrm{mg} / \mathrm{dl})\end{array}$ & $\begin{array}{c}\text { LDL } \\
(\mathrm{mg} / \mathrm{dl})\end{array}$ & $\begin{array}{c}\text { VLDL } \\
(\mathrm{mg} / \mathrm{dl})\end{array}$ \\
\hline Normal & $93 \pm 5$ & $60 \pm 4$ & $49 \pm 3.7$ & $33 \pm 3.8$ & $28 \pm 1.3$ \\
\hline Diabetic control & $224 \pm 8^{*}$ & $159.5 \pm 0.5^{*}$ & $31 \pm 1.4^{*}$ & $149 \pm 4.8^{*}$ & $66 \pm 4.4^{*}$ \\
\hline Gliclizide & $119 \pm 12^{\#}$ & $78 \pm 5.5^{* \#}$ & $46 \pm 1.9^{\#}$ & $61 \pm 10.9^{* \#}$ & $34 \pm 2.5^{\#}$ \\
\hline Ranolazine & $94 \pm 5.5^{\#}$ & $68 \pm 4.3^{\#}$ & $49 \pm 1^{\#}$ & $38 \pm 3.5^{\# \$}$ & $31 \pm 1.7^{\#}$ \\
\hline
\end{tabular}

*Statistically significant difference versus normal control group $(P$-value $<0.05)$ \# Statistically significant difference versus diabetic control group $(P$-value $<0.05)$

$\$$ Statistically significant difference versus gliclazide-treated group $(P$-value $<0.05)$ 
Figure 3: Effect of Gliclizide and Ranolazine on $\mathrm{HbA}_{1 \mathrm{c}}(\operatorname{serum} \%)$, AGEs (Mmol/l), HOMA (HOMA-RI Index) and NO (nmol/ml) in HFD and low dose STZ-induced diabetic rats:
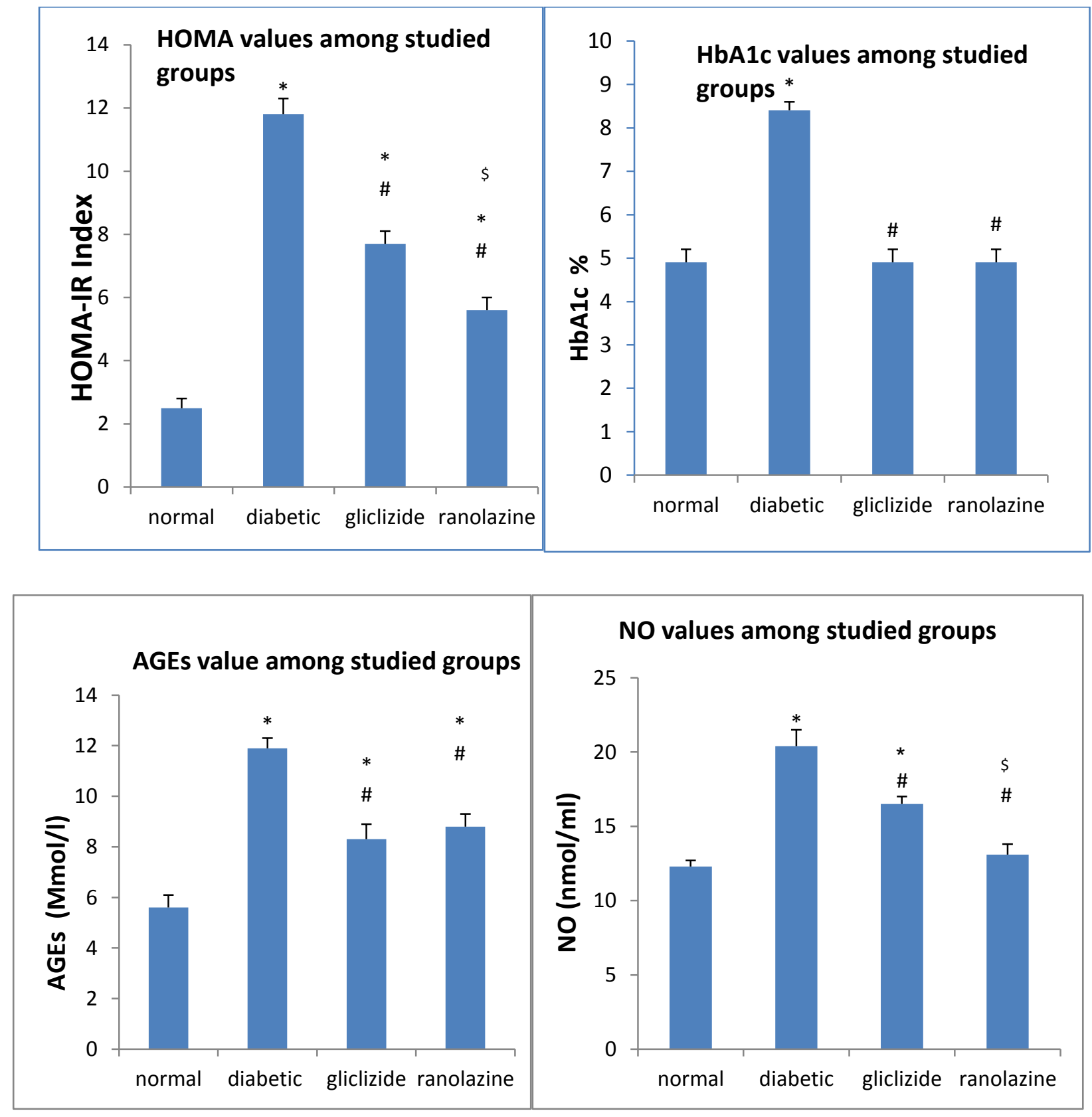

*Statistically significant difference versus normal control group (P-value $<0.05)$ \# Statistically significant difference versus diabetic control group $(P$-value $<0.05)$ $\$$ Statistically significant difference versus gliclazide-treated group $(P$-value $<0.05)$ 
Figure 4: Effect of Gliclizide and Ranolazine on oxidative stress markers (MDA (nmol/g), GSH (mg/g), SOD (U/g) and CAT (U/g)) in HFD and low dose STZ-induced diabetic rats:
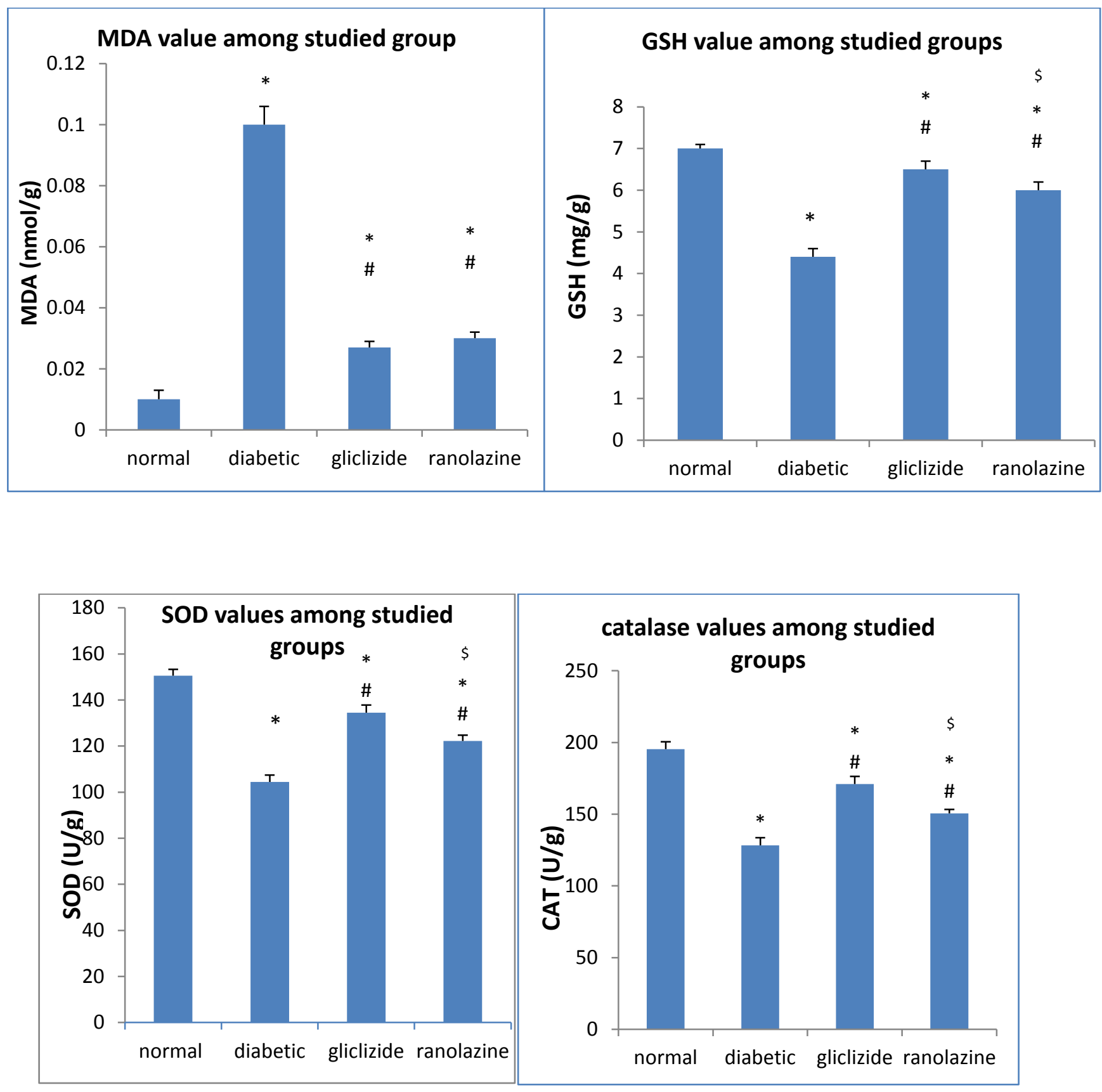

*Statistically significant difference versus normal control group (P-value $<0.05)$ \# Statistically significant difference versus diabetic control group $(P$-value $<0.05)$ $\$$ Statistically significant difference versus gliclazide-treated group $(P$-value $<0.05)$ 
Figure 5: Histopathological evaluation of pancreatic islets stained with H/E.
(A) Normal control group
(B)Diabetic control group (C)Gliclazide treated group
(D)Ranolazine treated group

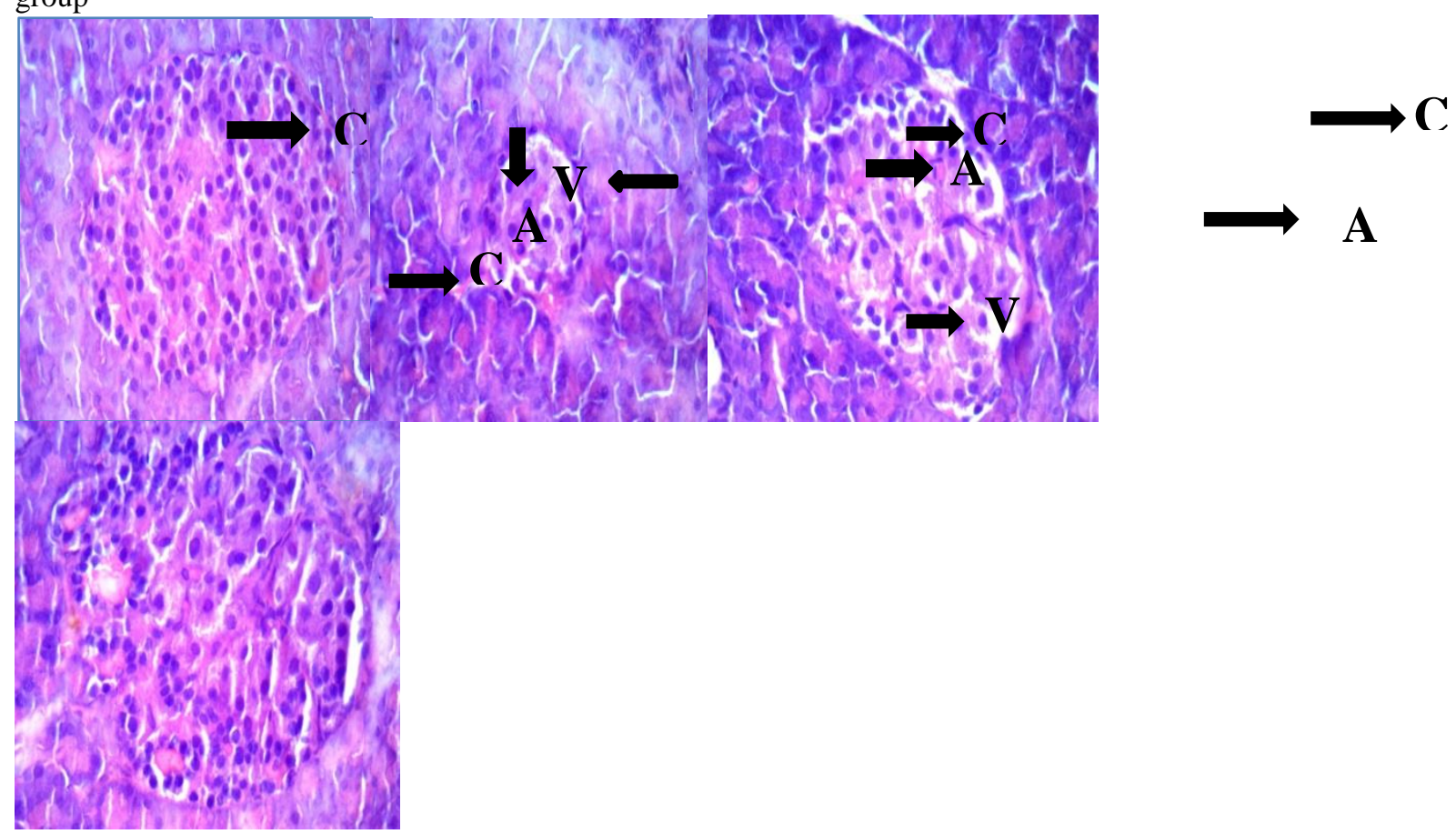

Fig. (5A-D) A photomicrograph of a section in pancreatic islets of a rat from all experimental groups (H\&EX400).

\section{Figure 6: Immunohistochemical staining of pancreatic islets for estimation of caspase-3.}

6(I)(A)Normal control group (B)Diabetic control group (C)Gliclazide treated group (D)Ranolazine treated group

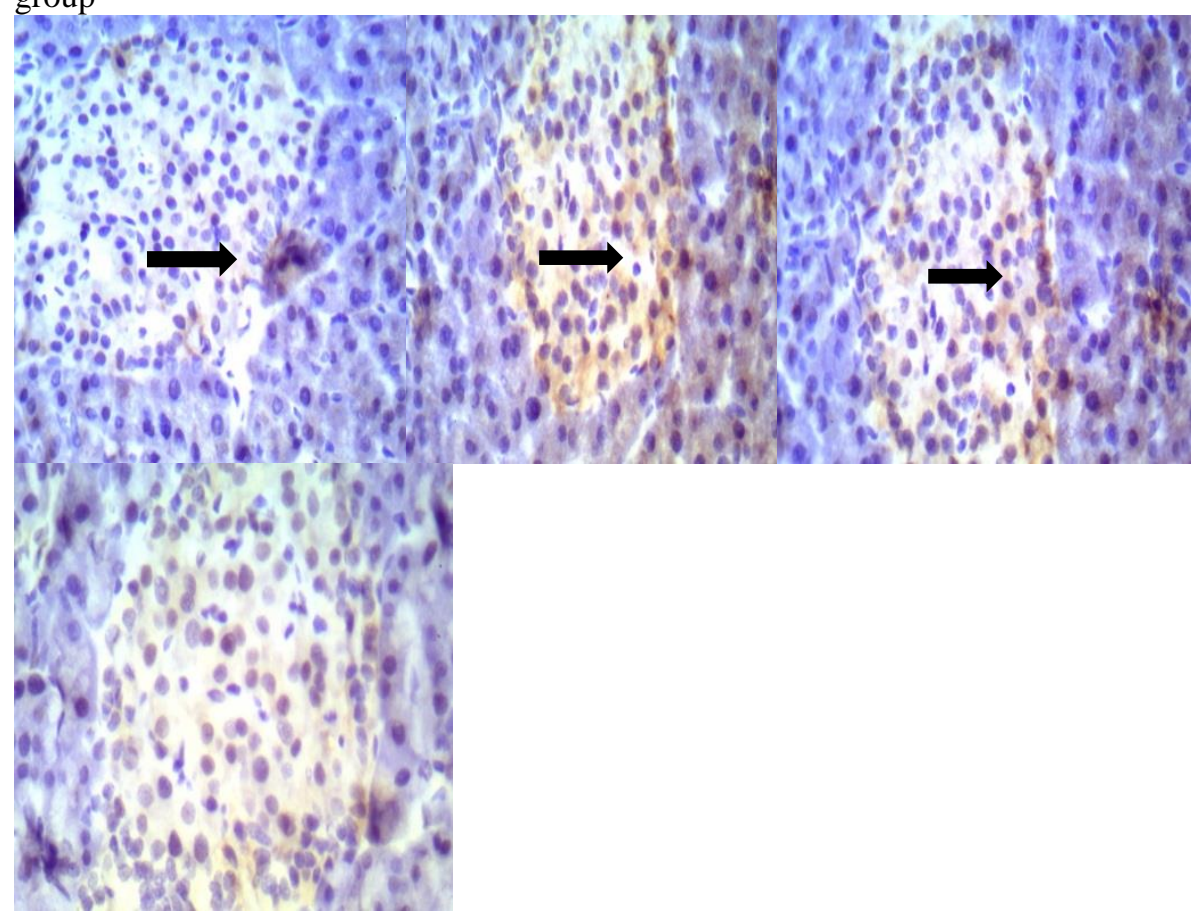

Fig. (6A-D) A photomicrograph of a section in pancreatic islets of a rat all experimental groups (Caspase-3 X 400). 
6(II) Estimation of caspase-3 marker in all experimental groups:

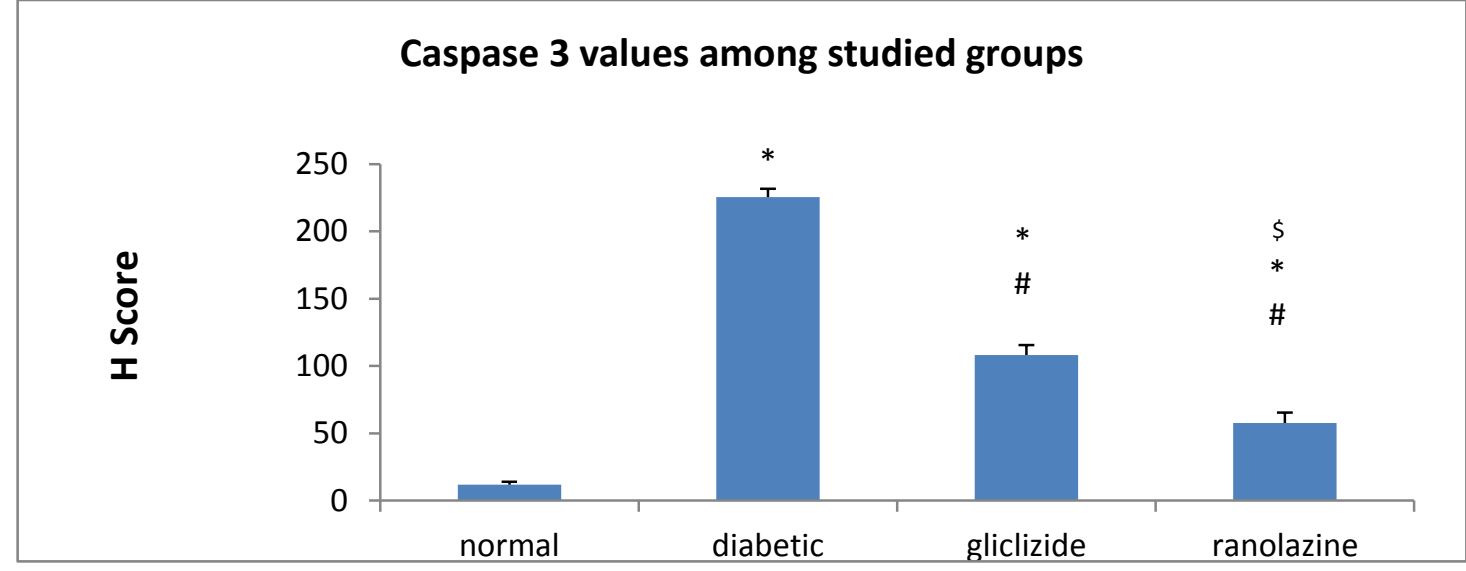

*Statistically significant difference versus normal control group $(P$-value $<0.05)$ \# Statistically significant difference versus diabetic control group $(P$-value $<0.05)$ $\$$ Statistically significant difference versus gliclazide-treated group (P-value $<0.05)$

\section{Discussion}

Ranolazine is a novel drug for angina which surges exercise duration and reduces frequency of anginal attacks in chronic angina patients. Ranolazine likewise has advantageous effects in diabetics as noticed by significant declines in $\mathrm{HbA}_{1 \mathrm{c}}$ in the clinical trials $[\mathbf{1 4 , 1 5}]$. This study was directed to assess the antidiabetic effect of ranolazine in HFD/STZ-challenged rats, which causes moderate hyperglycemia due to damage of pancreatic $\beta$-cells.

Streptozotocin (STZ) is a commonly powerful alkylating agent that yields a selective lethal effect on $\beta$-cells of pancreas and brings DM in most experimental animals. HFD followed by low dose STZ injection can trigger immune response and induce type $2 \mathrm{DM}$ as designated by the results of [16].

Gliclazide, which is used as a standard antidiabetic agent in the current study improved HFD/STZ-induced hyperglycemia via increasing insulin release from $\beta$-cells of pancreas and accelerating tissue uptake and consumption of glucose [17]. Ranolazine competently improved the harmful effects related to the HFD and low dose STZ as proven by reducing FBG level and $\mathrm{HbA}_{1 \mathrm{c}}[\mathbf{1 8}]$.

In accordance with [19] studies, the current study revealed that HFD/STZinduced hyperglycemia produced noticeable increased level of serum TG, TC 
and LDL- cholesterol (LDL-C) and decreased level of serum HDL cholesterol (HDL-C). One conceivable explanation could be afforded by [20] who highlighted that, this hyperlipidemia may be due to elevated level of cortisol and insulin insufficiency, which have an imperative role in fat accumulation process.

In agreement with [21], treatment with gliclazide proficiently lowered TC, TG and LDL-C concentrations and augmented HDL-C levels due to its stimulatory effect on the release of insulin. In accordance with [22,23], the current study shown that the HFD followed by low dose STZ produced hyperglycemia and hyperinsulinemia with subsequent surge in HOMA-IR index; that were competently ameliorated by treatment with either gliclazide or ranolazine.

Reactive oxygen species (ROS), nitric oxide, and peroxynitrite radicals, show an essential role in oxidative stress correlated to the pathogenesis of several important illnesses [24]. Vascular endothelial cells express iNOS in response to cytokines which produce NO that is involved in many inflammatory diseases [25]. Nitric oxide can react with ROS to produce vastly reactive oxidant species peroxynitrite; resulting in additional oxidative stress [26]. In accordance with the results of $[27,28]$ who highlighted that diabetes was linked to amplified expression of iNOS and elevated nitric oxide levels in STZ-induced diabetic rats, our results showed that HFD/STZ-challenged rats exhibited elevation in NO serum level. Treatment with either gliclizide or ranolazine reinstated the balance by lowering the generation of free radical and elevating the free radical scavenging; resulting in reduction of NO levels.

Advanced glycated end products (AGEs) are involved in endothelial dysfunction [29]. The augmented formation of AGEs establishes a possible mechanism of hyperglycaemia-induced diabetic complications [30]. In accordance with $[\mathbf{3 1 , 3 2}]$, our results revealed that gliclazide treatment 
competently lowered the augmented AGEs levels. Moreover, the current results revealed the antiglycation outcome of ranolazine on glucose-induced AGE formation.

High levels of free radicals can produce Products of Lipid peroxidation such as MDA which are significant in the pathogenesis of DM complication $[33,34]$. In the current study, a significant reduction in GSH, SOD and CAT and an elevation in MDA were observed in HFD/STZ-challenged rats. Treatment with either gliclazide or ranolazine produced a significant reduction in MDA and significant surge in GSH, SOD and CAT activity; reflecting the antioxidant characteristics of gliclazide and ranolazine. [35] revealed that gliclazide may lessen the oxidative stress via acting as a free radical scavenger [36]. Accordingly, the attained decreased expression of caspase- 3 is predictable and this outcome is in accordance with the results of [37] who reported that, the gliclazide's anti-apoptotic effect was linked to surge in protein kinase B level and a decline in caspase-3 levels. Treatment with ranolazine shows the same efficacy as that with gliclazide.

The histopathological analysis of the pancreas using H/E assured the biochemical markers and revealed the islets' collapse. In harmony with $[\mathbf{3 8 , 3 9}]$, the histopathological examinations of islets of pancreas in HFD/STZ-challenged rats showed hypocellularity, severe size shrinkage in association with deteriorating changes in pancreatic duct lining cells and significant surge in islet apoptosis rate in comparison to normal controls. These findings are confirmed by the immunohistochemical studies which revealed a surge in the apoptotic marker caspase-3.

In accordance with [40], the current study revealed that the administration of gliclazide to HFD/STZ-challenged rats significantly reduced the caspase-3 expression in pancreas. This finding supports the gliclazide's antioxidant role in 
mitigating the apoptosis in cells of pancreas. This could be explained by [41] who documented that, gliclizide could upregulate the antiapoptotic $\mathrm{Bcl} 2$ expression and downregulate the proapoptotic protein Bax expression.

The worsening of glycemic control in diabetes is supposed to be related to $\beta$-cell mass loss and progressive deterioration of $\beta$-cell function. The major finding of this study is that treatment with ranolazine decelerates the diabetes progression by reducing islets' apoptotic rate and preserving $\beta$-cell mass as proven by reduced caspase-3 level in HFD/STZ-challenged rats. Thus, the role of ranolazine in preservation of $\beta$-cell could be due to preservation of normal blood glucose level after HFD/STZ treatment. Another explanation could be afforded by [42] who stressed that ranolazine may apply advantageous effects on damage of beta cell, which is reliant on the cytosolic $\mathrm{Ca}^{2+}$. The cytosolic $\mathrm{Ca}^{2+}$ elevation surge antioxidatant enzymes and provide protection of beta-cells from the oxidative stress. Additionally, the augmented insulin sensitivity results in lowered blood glucose level and improvement of beta-cells environment.

In summary, the data from the current study reveals that ranolazine may apply possible ameliorating anti-diabetic effect by protecting $\beta$-cell mass and augmenting insulin sensetivity. In this setting, ranolazine has the potential to become a novel agent for treating patients with both diabetes and angina. Nevertheless, more pre-clinical studies are required to further describe the probable antidiabetic effect of ranolazine and its underlying molecular mechanisms. 


\section{Conclusion}

The results of this study showed that ranolazine improved diabetes by decreasing fasting blood glucose level, $\mathrm{HBA}_{1 \mathrm{c}}$, NO and AGEs. Moreover, it improved the oxidative stress markers, the histopathological picture of the pancreas and decreased the apoptosis.

\section{$\underline{\underline{\text { References }}}$}

[1] Wajchenberg BL (2007) beta-cell failure in diabetes and preservation by clinical treatment. Endocr Rev 28(2):187-218.

[2] Badran M. and Laher I (2012) Type II Diabetes Mellitus in ArabicSpeaking Countries. Int J Endocrinol 2012:1-11.

[3] Cannon CP (2008) Mixed dyslipidemia, metabolic syndrome, diabetes mellitus, and cardiovascular disease: clinical implications. Am J Cardiol 102(12A):5L-9L.

[4] Chaitman BR, Skettino SL, Parker JO, Hanley P, Meluzin J and et al (2004) Anti-ischemic effects and long-term survival during ranolazine monotherapy in patients with chronic severe angina. J Am Coll Cardiol 43(8):1375-82.

[5] Stone PH, Gratsiansky NA, Blokhin A, Huang IZ. and Meng L (2006) Antianginal Efficacy of Ranolazine When Added to Treatment With Amlodipine: The ERICA (Efficacy of Ranolazine in Chronic Angina) Trial. J Am Coll Cardiol 48(3):566-75.

[6] Chisholm JW, Goldfine AB, Dhalla AK, Braunwald E, Morrow DA and et al (2010) Effect of ranolazine on $\mathrm{A} 1 \mathrm{C}$ and glucose levels in hyperglycemic patients with non-ST elevation acute coronary syndrome. Diabetes Care 33(6):1163-8.

[7] Ning Y, Zhen W, Fu Z, Jiang J, Liu D and et al (2011) Ranolazine increases b-cell survival and improves glucose homeostasis in low-dose streptozotocin-induced diabetes in mice. J Pharmacol Exp Ther 337(1):50-8. 
[8] Fava D, Cassone-Faldetta M, Laurenti O, De Luca O, Ghiselli A. and et al (2002) Gliclazide improves anti-oxidant status and nitric oxide-mediated vasodilation in type 2 diabetes. Diabet Med 19(9):752-7.

[9] Srinivasan K, Viswanad B, Asrat L, Kaul CL. and Ramarao P (2005) Combination of high-fat diet-fed and low-dose streptozotocin-treated rat: A model for type 2 diabetes and pharmacological screening. Pharmacol Res 52(4):313-20.

[10] Lu HE, Jian CH, Chen SF, Chen TM, Lee ST and et al (2010) Hypoglycaemic effects of fermented mycelium of plaecilomyces farinosus (G30801) on high-fat fed rats with streptozotocin-induced diabetes. Indian J Med Res 131:696-701.

[11] Attia HN, Al-Rasheed NM, Al-Rasheed NM, Maklad YA, Ahmed AA and et al (2012) Protective effects of combined therapy of gliclazide with curcumin in experimental diabetic neuropathy in rats. Behav Pharmacol 23(2):153-61.

[12] Hennige AM, Burks DJ, Ozcan U, Kulkarni RN, Ye J and et al (2003) Upregulation of insulin receptor substrate-2 in pancreatic beta cells prevents diabetes. J Clin Invest 112(10):1521-32.

[13] Detre S, Saccani Jotti G. and Dowsett M (1995) A "quickscore" method for immunohistochemical semiquantitation: validation for oestrogen receptor in breast carcinomas. Clin Pathol 48:876-8.

[14] Timmis AD, Chaitman BR. and Crager M (2006) Effects of ranolazine on exercise tolerance and HbAlc in patients with chronic angina and diabetes. Eur Heart J 27(1):42-8.

[15] Scirica BM, Morrow DA, Hod H, Murphy SA, Belardinelli L and et al (2007) Effect of ranolazine, an antianginal agent with novel electrophysiological properties, on the incidence of arrhythmias in patients with non ST-segment elevation acute coronary syndrome: results from the metabolic efficiency with ranolazine for less ischemia in non ST-elevation acute coronary syndrome 
thrombolysis in myocardial infarction 36 (MERLIN-TIMI 36) randomized controlled trial. Circulation 116(15):1647-52.

[16] Papaccio G, Pisanti FA, Latronico MV, Ammendola E. and Galdieri M (2000) Multiple low dose and single high-dose treatments with streptozotocin do not generate nitric oxide. J Cell Biochem 77(1):82-91.

[17] Ojewole JA, Stephen O, Adewole SO. and Olayiwola G (2006) Hypoglycaemic and hypotensive effects of MomordicacharantiaLinn (Cucurbitaceae) whole-plant aqueous extract in rats. Cardiovasc J S Afr 17(5):227-32.

[18] Derr R, Garrett E, Stacy GA. and Saudek CD (2003) Is $\mathrm{HbA}(1 \mathrm{c})$ affected by glycemic instability? Diabetes Care 26(10):2728-33.

[19] Jurgonski A, Juskiewicz J. and Zdunczyk Z (2008) Ingestion of black chokeberry fruit extract leads to intestinal and systemic changes in a rat model of prediabetes and hyperlipidemia. Plant Foods Hum. Nutr 63(4):176-82.

[20] Hristova M. and Aloe L (2006) Metabolic syndrome - neuroirophic hypothesis. Med Hypotheses 66(3):545-9.

[21] Aquilante CL (2010) Sulfonylurea pharmacogenomics in Type 2 diabetes: the influence of drug target and diabetes risk polymorphisms. Expert Rev Cardiovasc Ther 8(3):359-72.

[22] Reed MJ, Meszaros K, Entes LJ, Claypool MD, Pinkett JG and et al (2000) A new rat model of type 2 diabetes: the fat-fed, streptozotocin-treated rat. Metabolism 49(11):1390-4.

[23] Zhang M, Lv XY, Li J, Xu ZG. and Chen L (2008) The characterization of high-fat diet and multiple low-dose streptozotocin induced type 2 diabetes rat model. Exp Diabetes Res 2008:704045.

[24] Shyur LF, Tsung JH, Chen JH, Chiu CY. and Lo CP (2005) Antioxidant properties of extracts from medicinal plants popularly used in Taiwan. Int $\mathbf{J}$ Appl Sci Eng 3(3):195-20. 
[25] Brinker AM, Ma J, Lipsky PE. and Raskin I (2007) Medicinal chemistry and pharmacology of genus Tripterygium (Celastraceae). Phytochemistry 68(6):732-66.

[26] Liorens S. and Nava E (2003) Cardiovascular diseases and the nitric oxide pathway. Curr Vasc Pharmacol 1(3):335-46.

[27] Stadler K, Jenei V, von Bölcsházy G, Somogyi A. and Jakus J (2003) Increased nitric oxide levels as an early sign of premature aging in diabetes. Free Radic Biol Med 35(10):1240-51.

[28] Madar Z, Kalet-Litman S. and Stark AH (2004) Inducible nitric oxide synthase activity and expression in liver and hepatocytes of diabetic rats. Pharmacology 73(2):106-12.

[29] Mashhoody T, Rastegar K. and Zal F (2014) Perindopril may improve the hippocampal reduced glutathione content in rats. Adv Pharm Bull 4(2):155-9. [30] Engelen L, Stehouwer CD. and Schalkwijk CG (2013) Current therapeutic interventions in the glycation pathway: evidence from clinical studies. Diabetes Obes Metab 15(8):677-89.

[31] Li W, Ota K, Nakamura J, Naruse K, Nakashima $E$ and et al (2008) Antiglycation effect of gliclazide on in vitro AGE formation from glucose and methylglyoxal. Exp Biol Med 233(2):176-9.

[32] Cardoso S, Santos RX, Correia SC, Carvalho C, Santos MS and et al (2013) Insulin-induced recurrent hypoglycemia exacerbates diabetic brain mitochondrial dysfunction and oxidative imbalance. Neurobiol Dis 49(1):1-12.

[33] Yazdanparast R, Ardestani A. and Jamshidi S (2007) Experimental diabetes treated with Achillea santolina: Effect on pancreatic oxidative parameters. J Ethnopharmacol 112(1):13-8.

[34] Halliwell B (2000) Lipid peroxidation, antioxidants and cardiovascular disease: how should we more forward? Cardiovasc Res 47(3):410-8. 
[35] Sliwinska A, Blasiak J, Kasznicki J. and Drzewoski J (2008) In vitro effect of gliclazide on DNA damage and repair in patients with type 2 diabetes mellitus (T2DM). Chem Biol Interact 173(3):159-65.

[36] Jennings PE (2000) Vascular benefits of gliclazide beyond glycemic control. Metabolism 49(10 Suppl 2):17-20.

[37] Li L. and Renier G (2009) The oral anti-diabetic agent, gliclazide, inhibits oxidized LDL-mediated LOX-1 expression, metalloproteinase-9 secretion and apoptosis in human aortic endothelial cells. Atherosclerosis 204(1):40-6.

[38] Adewole SO. and Ojewole JA (2007) Insulin-induced immuno histochemical and morphological changes in pancreatic beta-cells of streptozotocin-treated diabetic rats. Methods Find Exp Clin Pharmacol 29(7):447-55.

[39] Abo Gazia MM. and Hasan NM (2012) Effect of glabridin on the structure of ileum and pancreas in diabetic rats: A histological, immunohistochemical and ultrastructural study. Nat Sci 10 (3):78-90.

[40] Hanan A Soliman, Nadia A Eltablawy. and Mona S Hamed (2015) The ameliorative effect of Petroselinum crispum (parsley) on some diabetes complications. J Med Plants Stud 3(4):92-100.

[41] Del Guerra S, Grupillo M, Masini M, Lupi R, Bugliani M and et al (2007) Gliclazide protects human islet beta-cells from apoptosis induced by intermittent high glucose Diabetes Metab Res Rev 23(3):234-8.

[42] Drews G, Krippeit-DrewsP. and Dufer M (2010) Oxidative stress and beta-cell dysfunction. Pflugers Arch 460(4):703-18. 